\title{
Der onymische Artikel im Schweizerdeutschen in seiner Funktion als Genusmarker
}

\author{
Melanie Bösiger (Freiburg i. Ü.)
}

\begin{abstract}
In Swiss German dialects first names are commonly used with a preceding article. Historically, the function of these so-called onymic articles was to show the name's case. They first arose when inflection of first names had been omitted and case was thus no longer expressed in suffixes. The data gathered in 2016 in an online survey for the research project "Das Anna und ihr Hund" indicate that today the function of reflecting the case is hardly relevant. However, it is important to the speakers to emphasize the name's grammatical gender. The three grammatical genders in (Swiss) German are feminine, masculine, and neuter. Typically, the ononymic articles' grammatical gender corresponds to their referents' biological gender, i. e. feminine articles for women's names, masculine articles for men's names. But sometimes neuter articles are used with female or, less often, with male names. Therefore, the same first name can have different grammatical genders that are indicated with an onymic article, e.g. $d$ Anna (f.) or $s$ Anna (n.). The choice of the article depends on the speaker, the situation, the referent, and other factors. Based on these observations, it is argued that marking grammatical gender is the onymic article's main function and marking case is secondary.
\end{abstract}

\section{$1 \quad$ Einleitung}

De Claudias Ma isch mit em Gabi z Paris gsi.

Die kursiv gesetzten Ausdrücke sind Ausschnitte aus den Daten, die mit einer Online-Umfrage für das Projekt „Das Anna und ihr Hund“ erhoben wurden (nachfolgend: „Anna-Daten“), in dessen Zentrum der Verweis mittels neutralem Genus auf Frauen steht (cf. Kap. 3). ${ }^{1} \mathrm{Ob}$ ein Rufname im Neutrum oder im Femininum gebraucht wird, zeigt sich dabei am Artikel vor dem Namen. Historisch gesehen übernahm der onymische Artikel die Funktion der Kasusmarkierung, als im Schweizerdeutschen die Flexion der Vornamen aufgegeben worden ist (cf. Behagel 1923: 51f.). Der vorliegende Aufsatz geht hingegen der These nach, dass im heutigen Schweizerdeutschen die Markierung von Genus die primäre Funktion dieses onymischen Artikels geworden ist.

\footnotetext{
${ }^{1}$ Mehr zum Projekt in Christen/Baumgartner in diesem Band.

Linguistik online 107, 2/21 - http://dx.doi.org/10.13092/lo.107.7693
}

CC by 3.0 
Zuerst werden Aspekte der morphologischen Form und der Setzung bzw. Auslassung des onymischen Artikels anhand von Grammatiken und weiterer Forschungsliteratur zum Schweizerdeutschen theoretisch erarbeitet. Es geht dabei darum, die Funktion des Artikels in Bezug auf die Kasus- und Genusanzeige theoretisch darzulegen. Anschliessend werden die „Anna-Daten“ analysiert. Hier werden dieselben Schwerpunkte gesetzt: Zunächst wird die onymische Artikellosigkeit in den Daten herausgearbeitet, anschliessend werden die gesetzten Artikel und ihre Funktion untersucht. Von besonderer Bedeutung ist dabei die Analyse von genitivischen Possessivkonstruktionen (wie im einleitenden Beispiel de Claudias Ma), dem einzigen syntaktischen Ort, an dem Genitiv im Schweizerdeutschen noch einigermassen verbreitet ist (cf. Kap. 2).

\section{Der onymische Artikel im Schweizerdeutschen}

Bevor spezifisch auf die Funktion des onymischen Artikels eingegangen werden kann, soll das Artikelparadigma im Schweizerdeutschen dargestellt werden. ${ }^{2}$ Morphologisch gleiche Artikel in unterschiedlicher dialektaler Form werden auf einer Zeile angegeben, andere Artikel auf einer neuen. Dabei muss beachtet werden, dass der Genitiv im Schweizerdeutschen eine Sonderstellung hat: Er tritt ausser in einigen höchstalemannischen Regionen (cf. Fleischer/ Schallert 2011: 86) nicht mehr als freier Kasus auf, sondern kommt nur in festen Wendungen und in Possessivkonstruktionen mit Eigennamen oder als solchen gebrauchten Appellativen vor (cf. z. B. Fischer 1960: 190f., Meinherz 1920: 165, Henzen 1927: 179). Viele Sprecher_innen des Schweizerdeutschen brauchen gar keinen Genitiv. Wo im Folgenden von Genitiv die Rede ist, sind stets solche Possessivkonstruktionen gemeint.

\begin{tabular}{|l|l|l|l|}
\hline & feminin & neutral & maskulin \\
\hline Nominativ & $d, d i$ & $s, d s$ & $d r, d e$ \\
\hline Akkusativ & $d, d i$ & $s, d s$ & $d r, d e(? d e n)$ \\
\hline Dativ & $\begin{array}{l}d r, d e \\
e d e r / e r, a d e r / a r, i d e r / i r\end{array}$ & $\begin{array}{l}\text { em, dem, dum } \\
\text { im, am, um }\end{array}$ & $\begin{array}{l}\text { em, dem, dum } \\
\text { im, am, um }\end{array}$ \\
\hline Genitiv & $\begin{array}{l}s, d s \\
\text { der } d s^{3}\end{array}$ & $s, d s$ \\
\hline
\end{tabular}

Tabelle 1: Artikelparadigma im Schweizerdeutschen ${ }^{4}$

\footnotetext{
2 Die Angaben zu Nominativ, Dativ und Akkusativ sind dem Sprachatlas der deutschen Schweiz (SDS III, 131140) entnommen. Der Genitiv wird im SDS nicht thematisiert, die Angaben in der Tabelle sind eine Zusammenschau aus den vorliegenden Grammatiken zum Schweizerdeutschen: Untersucht wurden für diesen Aufsatz jene Bände der 20 Beiträge zur Schweizerdeutschen Grammatik (BSG), in denen verschiedene Autor_innen zwischen 1910 und 1941 die Grammatik in verschiedenen Regionen in der Deutschschweiz beschrieben haben, daneben Dauwalders (1992) Haslideutsche, Fischers (1960) Luzerndeutsche, Martis (1985) Berndeutsche, Suters (1992) Baseldeutsche und Webers (1948) Zürichdeutsche Grammatik sowie Hodlers (1969) Berndeutsche Syntax und ein handschriftliches Manuskript von Goldener (1908) zur Ostschweiz. Zusätzlich wurden die Grammatikteile in Schmutz/Haas' (2004) Senslerdeutschem und Schmid/Isslers (1982) Davoserdeutschem Wörterbuch analysiert.

${ }^{3}$ Die mit dem Maskulinum/Neutrum zusammenfallende Form $(d) s$ im Femininum kommt nur in possessiven Konstruktionen mit Eigennamen oder als solchen gebrauchten Appellativen vor.

${ }^{4}$ Im Folgenden wird jeweils eine Schreibweise herausgegriffen und im Text verwendet.
} 
Das Artikelparadigma zeigt, dass die Formen des Nominativs und Akkusativs zusammenfallen. ${ }^{5}$ Bei den Dativen kommen zwei verschiedene Arten von Artikeln vor: Zum einen die einfachen Dativartikel, im Neutrum und Maskulinum oftmals mit geschwundenem dentalem Anlaut (em), zum anderen können die Präpositionen ,in`oder , an` vor die Artikel treten (Präpositionale Dativmarkierung nach Seiler 2003: 14f.), was im Maskulinum und Neutrum (im, am, um) sowie teilweise im Femininum (ir, ar, er) zu einer Kontraktion des einfachen Dativartikels mit der den genannten Präpositionen führt. ${ }^{6}$ Im Genitiv kann die Form $s$ bzw. $d s$ für alle drei Genera gebraucht werden, selten wird noch unterschieden zwischen dem Neutrum/Maskulinum und einer eigenen femininen Form der. Die genitivischen Artikel werden in Kap. 2.2.1 genauer erläutert.

\subsection{Onymische Artikellosigkeit im Schweizerdeutschen}

Im Schweizerdeutschen gilt gemeinhin, dass der Artikel vor Rufnamen ,unmarkiert, ja obligatorisch[,] generalisiert und entpragmatisiert“" ist (Nübling et al. 2012: 125). In den Grammatiken zum Schweizerdeutschen und im Schweizerischen Idiotikon finden sich jedoch Ausnahmen von dieser Regel, sie sind hauptsächlich geographisch bedingt: Für das Berner Oberland, das Emmental, Deutschfreiburg und die östlichen Walserorte wird in der Forschungsliteratur Artikellosigkeit ausgewiesen (cf. Bucheli Berger 2006: 92; Hodler 1969: 33; Marti 1985: 80; Schmid/Issler 1982: XV; SDS III, 141; Idiotikon XIII, Sp. 1154-1159; für pragmatische Gründe der Artikellosigkeit cf. Kap. 2.2). Bucheli-Berger (2006: 93f.) zeigt anhand der Daten aus dem Syntaktischen Atlas der Schweiz (SADS), dass im Unterschied zu den Angaben im SDS kaum mehr Orte zu finden sind, an denen durchgehend Artikellosigkeit vorkommt. ${ }^{7}$ Hodler (1969: 33) beschreibt ebenfalls, dass die Setzung von Artikel neben die Artikellosigkeit trete. Hinweise darauf, dass bei der Artikelsetzung Unterschiede zwischen Nominativ, Dativ und Akkusativ bestehen, konnten in der Forschungsliteratur keine gefunden werden. ${ }^{8}$ Gesondert betrachtet werden muss indes der Genitiv, da bei ihm bezüglich onymischer Artikellosigkeit andere Regeln gelten als bei den übrigen Kasus, cf. dazu Kap. 2.2.1.

\footnotetext{
${ }^{5}$ Im Nhd. sind die nominativischen und akkusativischen Artikel im Femininum und Neutrum ebenfalls formal zusammengefallen, im Maskulinum bestehen mit $d e r$ und den noch eigene Formen. Für das Schweizerdeutsche wird maskulines den in der Forschungsliteratur selten ausgewiesen (cf. z. B: Hodler 1969: 357). Ob und wo es heute noch vorkommt, wird im Rahmen der im Entstehen begriffenen Dissertation der Verfasserin zum Thema Kasus im Schweizerdeutschen untersucht.

${ }^{6}$ Es lässt sich nicht immer eindeutig erkennen, ob eine Form als einfacher oder präpositional markierter Artikel zu verstehen ist. Insbesondere die Form em kann sowohl ein einfacher Artikel mit geschwundenem dentalem Anlaut sein oder eine Form mit in oder an, deren Vokal sich verändert hat (cf. Bösiger 2017: 70).

${ }^{7}$ Dies begründet Bucheli-Berger (2006: 93f.) unter anderem damit, dass für den SADS mehrere Personen pro Ort befragt wurden (für den SDS hingegen meist nur eine) und somit Variation innerhalb eines Ortes wahrscheinlicher ist.

${ }^{8}$ Schmuck/Szczepaniak (2014: 128f., 132) weisen in ihrer Untersuchung von Hexenverhörprotokollen aus Deutschland für mittel- und oberdeutsche Dialekte nach, dass der Artikel im Nominativ am seltensten, im Genitiv etwas häufiger und im Akkusativ und Dativ am meisten gebraucht wird. Für den Süden ihres Untersuchungsgebiets zeigen sie hingegen einen kasusunabhängigen häufigen Gebrauch des Artikels.
} 


\subsection{Zur Funktion des onymischen Artikels im Schweizerdeutschen}

Der Artikel hat in der deutschen Sprache verschiedene Funktionen: Er kann Kasus, Numerus, Genus, Definitheit und Markiertheit ausdrücken. Definitheit fällt als Funktion des onymischen Artikels weg, da „Eigennamen [...] als starre Designatoren per definitionem der (definit) unikalen Referenz dienen [...] und darum [...] eines Artikels zur Markierung dieser Art der Referenz nicht bedürfen“ (Kolde 1989: 92). Unbestimmter Artikel vor Rufnamen ist nur in wenigen Ausnahmefällen, in denen explizit die Unbekanntheit einer Person ausgedrückt werden soll, überhaupt möglich, so etwa im konstruierten Beispielsatz „Gestern hat eine Mireille angerufen“ (Nübling et al. 2012: 80f.). Ebenso stehen Rufnamen gemeinhin im Singular und nur in Ausnahmefällen wie „die zwei Annas unserer Klasse“ (Gallmann 1999: G2) im Plural, auch die Funktion des onymischen Artikels als Numerusmarker ist damit hinfällig. Für das Standarddeutsche kommt die Markiertheit ins Spiel. Es besteht eine Wahlfreiheit zwischen Artikelsetzung und Artikellosigkeit vor Rufnamen, die Setzung ist stets markiert (cf. Nübling et al. 2012: 125) und bringt ,eine stilistische Nuance zum Ausdruck [...], die je nach regionalsprachlicher Norm von ,vertraut" bis ,abwertend" reichen kann“ (Kolde 1995: 403). Schmuck/Szczepaniak (2014: 132) finden in Hexenverhörprotokollen aus dem 16. und 17. Jahrhundert bereits eine Süd-Nord-Entwicklung, bei der diese pragmatischen Faktoren der Artikelsetzung vor allem im Norden wirken, während der Artikel im Süden auch in „wertneutralen Kontexten“ gebraucht wird. Für das Schweizerdeutsche sind hingegen nur vereinzelte und wenig aussagekräftige Hinweise vorhanden, dass die Setzung des onymischen Artikels neben Artikellosigkeit vorkommt und eine pragmatische Funktion hat. So steht im Schweizerischen Idiotikon (XIII, Sp. 1194), dass Artikelsetzung und Artikelauslassung sich in „gewisse[n] Ausdruckswerte[n], die aber schwer zu fassen sind“, unterscheiden. Genauere Angaben zu diesen Ausdruckswerten finden sich höchstens bei Suter (1992: 110), der über das Baseldeutsche schreibt, dass die Setzung des Artikels ein „vertrauteres Verhältnis zwischen Nennendem und Genanntem“" schaffe. In seiner Grammatik gibt es im Folgenden aber keine Hinweise darauf, dass im Baseldeutschen Rufnamen ohne Artikel gebraucht werden. Ob er sich mit dieser Aussage auf den Unterschied zwischen Standard- und Schweizerdeutsch bezieht und im Schweizerdeutschen stets dieses vertrautere Verhältnis sieht, muss dahingestellt bleiben. Stärkere Hinweise darauf, dass der Artikel im Schweizerdeutschen pragmatische Markiertheit ausdrücke, gibt es keine. Es bleiben als Funktionen die Anzeige von Kasus und Genus.

\subsubsection{Der onymische Artikel als Kasusmarker}

Dass der Artikel vor Rufnamen deren Kasus markiert, lässt sich mit einem Blick in die Sprachgeschichte bestätigen: Während sich der Artikel in der deutschen Sprache seit dem Althochdeutschen ausgebreitet hat, sind Rufnamen lange Zeit artikellos geblieben. Der onymische Artikel ist ,gewissermaßen der letzte Entwicklungsschritt“ (Glaser 2008: 94). Insbesondere für die Frauennamen begründet Behaghel (1923: 49) die Artikellosigkeit damit, dass zwar ,feminine Singulare wegen ihrer mangelhaften Flexion in erhöhtem Maße des Artikels bedürfen“, was für weibliche Rufnamen aber nicht gelte, weil bei ihnen wie bei den männlichen Rufnamen eine Flexion bestand. Damit war die Kasusmarkierung am Namen selber gegeben und musste nicht in den Artikel ausgelagert werden. Behaghel (1923: 52) schreibt, dass die Artikel denn auch zuerst „,bei fremden Eigennamen [auftraten], nur in den obliquen 
Kasus 9 , aus Gründen der Deutlichkeit [...] in den Fällen, wo keine deutsche Flexionsendung möglich ist“. Diese Erklärung, dass „hergebrachte“ Rufnamen flektiert wurden und nur bei Frauennamen, ,die man als modern empfindet", ein Artikel gesetzt wurde, gibt auch Dauwalder (1992: 18) in seiner Grammatik zum Haslideutschen. Und Hodler (1969: 33) schreibt ebenfalls, dass der Artikel mit der Aufgabe der Flexion der Rufnamen zusammenhängt: Erst als am Rufnamen selber kein Kasus mehr ablesbar war, wurde der Artikel gesetzt. Sprachhistorisch gesehen ist der Artikel also ein Kasusmarker. Dies zeigt sich heute insbesondere noch an den Genitiv-Formen. Sie haben als einzige die Flexionsendung am Rufnamen behalten und sind durch die Endung $-S^{10}$ als Genitiv erkennbar, so zum Beispiel im Berndeutschen: Nom./Akk.: dr Walter, Dat.: em Walter, Gen.: Walters (cf. Hodler 1969: 33). Auf Artikelebene gibt es bei diesen Namen im Genitiv gemäss der Forschungsliteratur vier Möglichkeiten:

1. Selten wird heute noch zwischen maskulinem/neutralem Artikel $d s$ und femininem der unterschieden (cf. z. B. Baumgartner 1922: 139): ds Peters Jagge und der Lauras Mantel.

2. Häufiger hat sich der maskuline/neutrale Artikel $d s$ auch auf das Femininum ausgebreitet (cf. z. B. Brandstetter 1904: 42, Fischer 1960: 184): ds Peters Jagge und ds Lauras Mantel.

3. Gemäss Henzen (1927: 179) wird im Kanton Freiburg der Dativartikel verwendet, es ergeben sich Mischkonstruktionen in der Form von em Peters Jagge.

4. Von allen vier Kasus findet sich im Genitiv am häufigsten onymische Artikellosigkeit (cf. z. B. Hodler 1969: 52, Hotzenköcherle 1984: 231): Lauras Mantel. Letzteres passt insofern in die sprachhistorische Entwicklung, als dass der Artikel vor Rufnamen da gesetzt wird, wo er wegen Aufgabe der Namenflexion den Kasus markieren muss: Weil im Genitiv die Flexion am Namen bis heute besteht, ist der Artikel als Kasusmarker nicht notwendig und wird häufiger als in anderen Kasus weggelassen.

\subsubsection{Der onymische Artikel als Genusmarker}

Die These des vorliegenden Aufsatzes besagt, dass die primäre Funktion des onymischen Artikels im Schweizerdeutschen die Markierung von Genus (und eben nicht Kasus) sei. Einen ersten Hinweis darauf bietet der Sprachatlas der deutschen Schweiz (SDS). Auf der Karte zum Artikel vor männlichen Rufnamen (SDS III, 141) wird ersichtlich, dass im Berner Oberland und im Wallis der Rufname in seiner Vollform ohne Artikel gebraucht wird. In der Legende zur Karte wird Folgendes vermerkt (Ausz. im Original): „Im BE Oberland und im WS wird der Personenname gern im Diminutiv gebraucht und nimmt dann meist den (neutralen!) Artikel zu sich [...] (,Chrischte hät s gseit', aber ,ds Chrischti '). "11 Der SDS beinhaltet keine Karte zum Artikel vor Frauennamen, aber an dieser Karte zu den Männernamen lässt sich folgendes Schema ablesen: Die Vollform ist gemäss dem natürlichen Geschlechtsprinzip (cf. Köpcke/

\footnotetext{
${ }^{9}$ Historisch traten die Artikel gemäss Behaghel (1923: 53) zuerst in den obliquen Kasus auf. Der „heutige Stand“ bei denjenigen Mundarten, bei denen der Artikelgebrauch überhaupt einsetze, ist aber, dass der Artikel „bei heimischen wie fremden Namen in allen Kasus gesetzt wird“.

${ }^{10}$ Das Suffix -s, im Wallis als -sch realisiert, war ursprünglich den Maskulina und Neutra vorenthalten, hat sich aber auf Feminina und insbesondere auf Frauennamen ausgebreitet (cf. z. B. Kolde 1995: 403; Hodler 1969: 388; Bohnenberger 1913: 87). In den „Anna-Daten“ kommen keine schwach flektierten Rufnamen im Genitiv mehr vor, weswegen die schwache Flexion hier ausgeklammert und das -s-Suffix als einzige Genitiv-Markierung behandelt wird.

${ }^{11}$,Chrischte' ist die Schweizerdeutsche Vollform von Christian, ,Chrischti' ist die diminuierte Form davon.
} 
Zubin 1984: 28) als Maskulinum zu verstehen. Das grammatische Genus stimmt mit dem biologischen Sexus des Trägers überein und wird darum nicht explizit ausgedrückt. Beim Diminutiv wird hingegen die fehlende Korrelation zwischen neutralem Genus und männlichem Sexus durch die Setzung des Artikels gekennzeichnet. Dass dort, wo der onymische Artikel und Artikellosigkeit nebeneinander vorkommen, der nicht gesetzte Artikel auf Genus-SexusKongruenz zwischen Rufname und Träger_in verweist, während die Setzung des Artikels eine Genus-Sexus-Inkongruenz ausdrückt, lässt sich mit zwei in der Forschungsliteratur aufgeführten Tendenzen stützen: Zum einen lässt sich herauslesen, dass Männernamen häufiger ohne Artikel vorkommen als Frauennamen (cf. z. B. Stucki 1917: 283). So sind im Schweizerischen Idiotikon (XIII, Sp. 1154-1194) mehr Belege für artikellose Männer- als Frauennamen vorhanden, ebenso verhält es sich auf der entsprechenden Karte im Atlas zur deutschen Alltagssprache (cf. Elspaß/Möller 2012). Zum anderen wird in einigen Grammatiken darauf hingewiesen, dass ,alle weiblichen Eigennamen grammatisch Neutra [sind], und zwar in normaler wie in diminuierter Form“ (Marti 1985: 81), oder dass zumindest unter gewissen Bedingungen Frauennamen als Neutra gebraucht werden (cf. z. B. Suter 1992: 69). Kombiniert man diese beiden Tendenzen, ergibt sich wiederum dasselbe Schema: Bei Männernamen ist das maskuline Genus als Default gesetzt und es wird davon abgesehen, das mit dem Sexus kongruente Genus explizit zu markieren (nur Diminutive werden eben mit Artikel als Neutra markiert) - bei Frauennamen hingegen wird der onymische Artikel gesetzt, weil das vom Sexus abweichende neutrale Genus entweder durchgehend verwendet wird oder zumindest möglich ist, was eine Genusmarkierung notwendig macht.

\section{Die „Anna-Daten“"}

Die Ergebnisse im vorliegenden Beitrag basieren auf Daten, die für das Projekt „,Das Anna und ihr Hund“" erhoben worden sind, das unter anderem das am onymischen Artikel ausgedrückte Genus von Frauennamen in den Vordergrund stellt. Die Daten entstammen einer OnlineUmfrage, die im Rahmen des Projekts zwischen Januar und Juni 2016 durchgeführt wurde. Es sind insgesamt 1'747 gültige Antworten eingegangen. Sie stammen von Personen aus allen Altersklassen (,jünger als 20 Jahre' bis ,80 Jahre oder älter') ${ }^{12}$ aus der gesamten Deutschschweiz, 90 Prozent der Teilnehmenden waren weiblich ${ }^{13}$. Für den vorliegenden Aufsatz wurden Daten aus zwei verschiedenen Fragetypen analysiert: zum einen aus Fragen mit freiem Antwortfeld (Umschreibung einer Situation mit einer im Dialekt zu beantwortenden Frage, für die Erhebung des Genitivs eine Übersetzung) sowie Ankreuzaufgaben, bei denen die Teilnehmenden jene vorgegebenen Sätze ankreuzen sollten, die sie in ihrem Dialekt sagen können.

\footnotetext{
12 Dabei wurden Kategorien in Zehnjahresschritten angelegt. Aus den Kategorien ,20-29 Jahre` bis ,60-p69 Jahre` sind ungefähr gleich viele Antworten vorhanden, bei jüngeren und älteren sind weniger, aber trotzdem noch einige Datensätze auswertbar, siehe für die genaue Verteilung Bösiger (2017: 17).

13 Wegen diesem hohen Frauenanteil wird im Folgenden beim Bezug auf Teilnehmende, wenn keine geschlechterneutrale Form möglich ist, das generische Femininum verwendet. Das Geschlecht der Teilnehmenden wurde für die vorliegenden Auswertungen nicht berücksichtigt. Wird von einer ,Teilnehmerin` als ,sie‘ gesprochen, muss dies nicht heissen, dass es sich um eine Frau handelt.
} 


\subsection{Onymische Artikellosigkeit}

In der Forschungsliteratur wird artikellose Verwendung von Rufnamen nur für einige Regionen ausgewiesen (cf. Kap. 2.1) und scheint zudem rückläufig zu sein. Die „Anna-Daten“ zeigen aber ein ganz anderes Bild: Artikellose Rufnamen sind in der gesamten Deutschschweiz belegt. Dabei muss beachtet werden, dass der feminine Artikel $d$ im Schweizerdeutschen keinen Vokal und damit keinen Silbenwert hat und daher in der gesprochenen Sprache vor einigen Anlauten von Assimilation betroffen ist. So wird laut Meyer (1967: 31) der Artikel in $d$ Mirka verändert gesprochen als pMirka, bei $d$ Beatrice führt die Assimilation zu einer Fortisierung des Anlauts zu Peatrice und bei $d$ Tina ist der Artikel im gesprochenen Tina gar nicht mehr zu hören. Die Verschriftung dieser phonetischen Assimilation kann dazu führen, dass der Artikel nicht geschrieben wird. ${ }^{14}$ Das Bild der sich über die gesamte Deutschschweiz ausbreitenden Artikellosigkeit bleibt aber auch dann bestehen, wenn nur diejenigen Namen aus den „Anna-Daten“ berücksichtigt werden, bei denen der Anlaut keine Assimilation zulässt (cf. Bösiger 2017: 2939).

\subsection{Der onymische Artikel im Genitiv}

Der Genitiv wurde abgefragt mit einer Übersetzungsaufgabe, in der die possessiven Konstruktionen ,Annas Teddybär', ,Vrenis Haus', ,Margrits Katze‘ und ,Claudias Mann' in den Dialekt übertragen werden mussten. Der Genitiv hat im Schweizerdeutschen wie oben erwähnt schon darum eine Sonderstellung, weil er nur noch selten vorkommt. Zudem wurde bereits gezeigt, dass Rufnamen im Genitiv die Flexions-Endung $-s$ behalten haben, was einen Gebrauch ohne onymischen Artikel begünstigt. ${ }^{15}$ Wie in Kap. 2.2.1 beschrieben, kommt auf Artikelebene im Genitiv neben der Artikellosigkeit die alte Unterscheidung von maskulinem/neutralem $d s$ und femininem der vor, dies aber nur selten und $d s$ hat sich weitgehend auf alle drei Genera ausgebreitet. Daraus folgt, dass ausser bei den seltenen Fällen mit altem femininem der am Genitiv kein Genus ablesbar ist.

Genau hier zeigt sich in den „Anna-Daten“, dass einigen Teilnehmenden mit dieser Genusnivellierung offenbar nicht wohl ist. ${ }^{16}$ Sie wollen sie anscheinend vermeiden und wenden verschiedene Strategien an, um Rufnamen in possessiven Genitivkonstruktionen wieder ein Genus zuzuweisen. Diese basieren auf der Übernahme eines Artikels aus anderen Kasus, der eine eindeutige Genusmarkierung zulässt. Zum einen wird der Dativartikel in den Genitiv übernommen, was in der Forschungsliteratur nur in Freiburg nachgewiesen ist (cf. Kap. 2.2.1). Gemäss den „Anna-Daten“ kommen aber auch im (angrenzenden) Kanton Bern Dativartikel in

\footnotetext{
${ }^{14}$ Hinweise auf dieses Nicht-Schreiben von assimilierten Artikeln geben unter anderem jene Antworten, in denen der Artikel in Klammern gesetzt wurde, so von A1208: „(d) Maria“, C574: „,(d) Jessica“ oder B121: „Das isch (d) Beatrice [...]“, cf. Bösiger (2017: 33).

15 Die „Anna-Daten“ zeigen allgemein eine Unsicherheit seitens der Teilnehmenden, was die Bildung des Genitivs angeht. Es lässt sich eine Vielzahl verschiedener Formen erkennen, von denen einige keine eindeutigen Genitive sind. Beschrieben werden hier darum nur jene Formen, die durch die $-s$ - bzw. $-s c h$-Suffigierung am Rufnamen eindeutig als Genitive zu werten sind.

16 Die Teilnehmenden haben für die Antworten schriftliche Übersetzungen angegeben. Inwiefern die Schriftlichkeit und die Vorgabe des standarddeutschen Genitivs dabei einen Einfluss auf die Wahl des Artikels in der schweizerdeutschen Antwort hat, kann mit den vorliegenden Daten nicht untersucht werden.
} 
Genitivkonstruktionen vor. Bei femininen Formen kann dabei nicht zwischen altem Genitiv mit der und Genitiv mit dativischem Artikel, der ebenfalls der lautet, unterschieden werden. Die Unterscheidung ergibt sich erst dann, wenn von einer Teilnehmerin Belege für feminine und maskuline bzw. neutrale Formen vorliegen. Dort zeigt sich, ob bei den Maskulina/Neutra der rein genitivische Artikel $d s$ gesetzt wird, oder ob das dativische em gewählt wird. Daraus lässt sich schliessen, als welcher Kasus der zu verstehen ist. In den „Anna-Daten“ wurden nur Frauennamen abgefragt. Die Frage war aber so gestaltet, dass vor allem wegen der morphologischen Gestalt des Namens (Vreni als Diminutiv, cf. Baumgartner/Christen 2017: 125) oder auch wegen unterschiedlichem suggeriertem Alter der bezeichneten Personen (Anna mit dem Teddybär als Kind, Margrit als Name, den vorwiegend ältere Frauen tragen) möglicherweise bei einigen Namen der neutrale Artikel elizitiert wurde. Somit liegen von vielen Teilnehmenden sowohl Bildungen mit femininem als auch mit neutralem Artikel vor. Wenn ein Artikel, zum Beispiel bei em Vrenis Huus, eindeutig im Dativ steht, darf dieser Kasus auch bei den femininen Artikeln vor den anderen Namen (der Margrits Chatz) angenommen werden. Gleichzeitig bleibt der Name durch das Suffix $-s$ eindeutig als Genitiv markiert, es ergibt sich hier auf Kasusebene eine Mischkonstruktion (Dativartikel und Genitiv-Endung), die dafür aber in ihrem Genus eindeutig ist - was bei der rein genitivischen Form ds Vrenis Huus bzw. ds Margrits Chatz etc. nicht der Fall wäre.

Die „Anna-Daten“ zeigen zudem sechs Datensätze, die in possessiven Genitiv-Konstruktionen den aus dem Nominativ/Akkusativ stammenden femininen Artikel $d$ aufweisen, wie die folgende Tabelle zeigt. Auffällig ist, dass alle Belege aus dem Höchtalemannsichen stammen, also aus jenem Gebiet, in dem der Genitiv lebendiger ist als anderswo. Sie stammen somit von Sprecher_innen, denen Genitivkonstruktionen vertraut sind.

\begin{tabular}{|c|c|c|c|c|c|}
\hline $\begin{array}{l}\text { Teilnehme- } \\
\text { rin }\end{array}$ & Ort & $\begin{array}{l}\text { Annas } \\
\text { Teddybär }\end{array}$ & Vrenis Haus & $\begin{array}{l}\text { Margrits } \\
\text { Katze }\end{array}$ & $\begin{array}{l}\text { Claudias } \\
\text { Mann }\end{array}$ \\
\hline A817 & $\begin{array}{l}\text { nicht lokali- } \\
\text { siert }\end{array}$ & Anna's Teddybär & Vreni's Hus & $\begin{array}{l}\text { d'Margrits } \\
\text { Katz }\end{array}$ & Claudias Maa \\
\hline $\mathrm{C} 227$ & Jaun FR & D’Annas Baer & $\begin{array}{l}\text { ts' Vrenes } \\
\text { Huus }\end{array}$ & $\begin{array}{l}\text { ts' Margrits } \\
\text { Chatz }\end{array}$ & d'Claudias $M o$ \\
\hline $\mathrm{C} 365$ & Gstaad BE & $\begin{array}{l}d \text { Annas } \\
\text { Teddybär }\end{array}$ & ds Vrenis Hus & $\begin{array}{l}\text { dMargrits } \\
\text { Chatz }\end{array}$ & Cloudias Maa \\
\hline D105 & Glis VS & - & $\begin{array}{l}\text { VVrenisch } \\
\text { Hüs }\end{array}$ & $\begin{array}{l}\text { Margritschs } \\
\text { Chatz }\end{array}$ & $\begin{array}{l}\text { Claudiasch } \\
\text { Maa }\end{array}$ \\
\hline D144 & Gruben VS & $\begin{array}{l}\text { Annasch Teddy- } \\
\text { bär, z'Annasch } \\
\text { Teddybär }\end{array}$ & $\begin{array}{l}\text { Z’Vrenisch } \\
\text { Hüs }\end{array}$ & $\begin{array}{l}\text { D'Margritsch } \\
\text { Chatz }\end{array}$ & $\begin{array}{l}\text { Z'Claudiasch } \\
\mathrm{Ma}\end{array}$ \\
\hline D146 & $\begin{array}{l}\text { Gasenried } \\
\text { VS }\end{array}$ & $\begin{array}{l}\text { Ds Annasch } \\
\text { Teddybär }\end{array}$ & $\begin{array}{l}\text { D Vernisch } \\
\text { Hüüs }\end{array}$ & $\begin{array}{l}\text { D Margritsch } \\
\text { Chatza }\end{array}$ & $\begin{array}{l}\text { Claudiasch } \\
\text { Mannji }\end{array}$ \\
\hline
\end{tabular}

Tabelle 2: Genitiv-Konstruktionen mit dem Artikel d (jeweils kursiv hervorgehoben, zum Vergleich andere Genitiv-Konstruktionen derselben Teilnehmenden) ${ }^{17}$

\footnotetext{
${ }^{17}$ Die Angaben in der Tabelle wurden von den Teilnehmenden genau so gegeben, allfällige Tippfehler u. ä. wurden nicht korrigiert.
} 
Mit Bezug auf Bösiger (2017: 29-37) muss hier angemerkt werden, dass bei Claudia ein Sonderfall vorliegt: Weil hier der feminine Artikel $d$ vollständig assimilieren kann, kommt es wohl vor, dass Teilnehmende ihn zwar ,meinen', aber nicht schreiben, und der Name darum artikellos gebraucht scheint. In der gesprochenen Sprache kann der Artikel auch vor Margrit durch Assimilation verändert werden, was ebenfalls zu einer Nicht-Schreibung des Artikels führen kann (cf. Kap. 3.1). So darf bei C365, D146 und D105 (wo der Genitiv von Anna fehlt) vermutet werden, dass ihre artikellosen Nennungen von Claudia und Margrit als feminine Artikel $(d)$ gemeint sind. Mit diesen dreien sowie C227 und D144, die bei allen vier Namen entsprechende Artikel schreiben, zeigt die Tabelle fünf Datensätze, die durchgehend die Opposition ,neutrales $d s$ vs. feminines $d$ ' verwenden. Warum A817 vor Margrit den nominativischen/akkusativischen Artikel schreibt, vor die anderen Namen aber keinen Artikel setzt, muss offen bleiben. Die Assimilation kommt hier als Begründung nicht infrage, sie hat vor Anna keinen Einfluss auf den Artikel.

Die genannten Teilnehmenden bilden also - wie auch bei der Übernahme des Dativ-Artikels Konstruktionen, die im Kasus uneindeutig sind, dafür ein am Artikel eindeutig erkennbares Genus tragen. Aber warum wird der Artikel aus dem Nominativ/Akkusativ in den Genitiv übernommen? Im Folgenden soll ein Erklärungsversuch dargelegt werden. Im Nominativ können Frauennamen im Femininum mit dem Artikel $d$ oder im Neutrum mit dem Artikel $d s$ verwendet werden. Im Genitiv gilt dagegen gemeinhin, dass $d s$ für alle drei Genera gesetzt wird. Weil der Genitiv aber sehr selten und (fast) nur noch mit Eigennamen vorkommt, ist sein Artikel einigen Teilnehmenden nicht vertraut. Im Neutrum fällt ferner der Artikel im Nominativ/Akkusativ mit demjenigen im Genitiv zusammen: Nom./Akk. ds Vreni, Gen. $d s$ Vrenis Huus. Es scheint, als ob einige Teilnehmende das genitivische $d s$ darum als Neutrum reanalysieren. Nun wollen sie scheinbar nicht oder nicht bei allen Rufnamen das Neutrum verwenden, gelangen aber auf der Suche nach einem im Genus eindeutig femininen Artikel nicht zum alten genitivischen der zurück - was überdies auch keine rein feminine Form ist, sondern mit dem maskulinen Nominativ-/Akkusativartikel zusammenfällt -, sondern übernehmen die dem Nominativ/Akkusativ entsprechende feminine Form $d$. Es folgt also aus der Beobachtung, dass das nominativisch/akkusativische Neutrum mit $d s$ einen Genitiv mit $d s$ nach sich zieht, die Annahme, dass folglich nominativisch/akkusativisches Femininum mit $d$ auch einen Genitiv mit $d$ ergebe: Nom./Akk. $d$ Margrit, Gen.: $d$ Margrits Chatz. Das folgende Schema stellt die Übernahme des Nominativ-/Akkusativartikels in den Genitiv noch einmal dar:

$\begin{array}{lll}\text { Beobachtung beim neutralen Diminutiv: } & \text { Nom./Akk. } & \text { Gen. } \\ \text { Übernahme bei der femininen Vollform: } & \boldsymbol{d} \text { Vreni } & \boldsymbol{d} \text { S Vrenis Hus } \\ & \boldsymbol{d} \text { Margrit } & \rightarrow \boldsymbol{d} \text { Margrits Chatz }\end{array}$

Die These, dass $d s$ im Genitiv als Neutrum interpretiert wird, lässt sich durch eine weitere Beobachtung in den „Anna-Daten“ stützen. Die Teilnehmenden konnten bei der Übersetzung der Possessivkonstruktionen wie Margrits Katze aus dem Standarddeutschen in ihren Dialekt neben dem Genitiv auch dativische Bildungen mit ,von' ( $d$ Chatz vo de Margrit) oder mit Possessivpronomen (de Margrit ihri Chatz) verwenden. Nur rund ein Drittel der Teilnehmenden haben überhaupt Genitive verwendet - und dies nicht durchgehend bei allen Namen. Die folgende Tabelle zeigt, welcher Vorame wie oft im Genitiv vorkommt und mit welchen Artikeln die einzelnen Namen gebraucht werden (cf. Bösiger 2017: 65): 


\begin{tabular}{|l|l|l|l|l|}
\hline Artikel / Name & Anna & Claudia & Margrit & Vreni \\
\hline $\boldsymbol{o}$ & 242 & 225 & 214 & 234 \\
\hline $\boldsymbol{d} \boldsymbol{d e r}$ & 58 & 77 & 74 & $104^{*}$ \\
\hline $\boldsymbol{a m} / \boldsymbol{e m} / \mathbf{i m}$ & 68 & 63 & 52 & $5^{*}$ \\
\hline $\boldsymbol{a r} / \boldsymbol{e r} / \boldsymbol{i r}$ bzw. $\boldsymbol{a}$ der/e der/i $\boldsymbol{d e r}$ & 3 & 2 & 14 & $84^{*}$ \\
\hline $\boldsymbol{d}$ & 20 & 21 & 24 & $1^{*}$ \\
\hline nicht kategorisierbar & 2 & 1 & 7 & 2 \\
\hline Total & 0 & 0 & 0 & 2 \\
\hline
\end{tabular}

Tabelle 3: Genitive sortiert nach Namen und verwendetem Artikel; mit * markiert jeweils stark von den anderen Namen abweichende Werte.

Vreni als einzige Form im Diminutiv ist offensichtlich am anfälligsten für das Neutrum und wird darum häufiger mit dem Artikel am/em/im vorwendet als mit femininem der bzw. ar/er/ir. Besonders auffällig ist dabei auch, dass ebendieser Name mit 430 Nennungen deutlich am häufigsten in den Genitiv gesetzt wird, während die drei anderen Namen 385, 389 und $393 \mathrm{Mal}$ und damit alle drei praktisch gleich oft im Genitiv gebraucht werden. Die Analyse der verwendeten Artikel zeigt, dass bei Vreni insbesondere mehr Genitive mit dem Artikel $d s$ vorkommen als bei den anderen Namen. Hier lässt sich eine andere Verhaltensweise herauslesen bei Teilnehmenden, die sich am vermeintlich neutralen Artikel vor eigentlich feminin gebrauchten Rufnamen zu stören scheinen: Statt wie die oben genannten Teilnehmenden auf Artikel aus den anderen Kasus zurückzugreifen, die eine Unterscheidung zwischen Neutrum/Maskulinum und Femininum erlauben, vermeiden sie schlicht den Genitiv bei Rufnamen, die sie feminin verwenden, und bilden ihn nur bei jenen, die sie ins Neutrum setzen (hier also ds Vrenis Huus).

\subsection{Der onymische Artikel im Dativ}

In den „Anna-Daten“ kommen im Dativ sehr viel weniger artikellose Varianten vor als in den anderen Kasus. Die Belege finden sich ausserdem fast nur in dem in der Forschungsliteratur ausgewiesenen artikellosen Gebiet (cf. Kap. 2.1), von einer Ausbreitung der Artikellosigkeit in andere Gebiete kann beim Dativ kaum gesprochen werden. Die Daten zeigen allesamt keine freien Dative (cf. Fleischer/Schallert 2011: 96f.): Einerseits wird eine Ferienreise mit Gabi nach Paris beschrieben und die Teilnehmenden haben die Frage beantwortet, mit wem sie in Paris gewesen seien. Die Antworten sollten in einer Formulierung wie mit de Gabi (bin ich z Paris gsi) gegeben werden, also mit einer Präposition und einem Dativ. Andererseits konnten wie oben erläutert bei den Possessivkonstruktionen die zwei dativischen Bildungen mit der Präposition ,von' ( $d$ Chatz vo de Margrit) oder mit Possessivpronomen (de Margrit ihri Chatz) genannt werden. Bei den Konstruktionen mit Possessivpronomen kommen auch präpositional markierte Dative mit den Präpositionen an und in vor (a/i de Margrit ihri Chatz, cf. Kap. 2). Gemäss Seiler (2003: 68) sind diese Präpositionen vor dem Artikel als „Verstärkung [...] des Dativs“ zu verstehen - umgekehrt könnte man also sagen, dass der Artikel in seiner Funktion als Kasusmarker an diesen Stellen geschwächt wird, weil bereits die Präposition den Dativ einfordert. Dies beobachtete bereits Behaghel (1923: 52): Rufnamen nach einer Präposition stehen „,in älterer Zeit meist ohne Artikel, dann wird der Artikel häufig, ist aber etwa seit 
Schiller wieder geschwunden, denn die Präposition läßt den Kasus deutlich genug erkennen““ Dies gilt auch nach den Präpositionen von und mit bei den anderen Dativen aus den „AnnaDaten“. Wenn der Artikel also primär Kasus anzeigen würde, müsste man annehmen, dass er nach Präpositionen als erstes verschwindet, weil er dort als Kasusmarker ohnehin geschwächt ist. Wie die „Anna-Daten“ aber zeigen, ist genau das Umgekehrte der Fall: Nach Präpositionen ist keine zunehmende Artikellosigkeit festzustellen, insbesondere bei den präpositional markierten Dativen kommt gar keine Artikellosigkeit vor.

\section{$4 \quad$ Fazit und Ausblick}

Die in diesem Aufsatz beschriebenen Ergebnisse zeigen, dass sich die Funktion des onymischen Artikels im Schweizerdeutschen gewandelt hat. Ursprünglich war der Artikel ein Kasusmarker, der die wegfallenden Flexionsendungen an den Rufnamen ersetzte. Dass die Funktion der Kasusanzeige heute sekundär geworden ist, zeigen vor allem die im Folgenden noch einmal zusammengefassten Erkenntnisse aus den „Anna-Daten“:

1. Eine Kasusunterscheidung mit dem Artikel zwischen Nominativ und Akkusativ ist gar nicht möglich, da seine Formen in den beiden Kasus bei allen drei Genera zusammengefallen sind.

2. Bei den Genitiv-Konstruktionen zeigen sich Verschiebungen zwischen den Kasus, indem bei possessiven Genitiven Artikel aus dem Dativ oder aus dem Nominativ/Akkusativ verwendet werden. So vermischen sich zwei Kasus in einer Konstruktion.

3. Es hat sich gezeigt, dass der Artikel im Nominativ und Akkusativ zusehends verschwindet (und der Genitiv ebenfalls ohne Artikel gebildet wird), im Dativ nach Präpositionen aber kaum ausgelassen wird. Gerade da, wo der Dativ durch die Präposition gestärkt und der Artikel als Kasusanzeiger somit geschwächt ist, - wo seine Auslassung im Bezug auf die Kasusmarkierung also die kleinsten Konsequenzen hätte - wird er nicht weggelassen.

All dies deutet darauf hin, dass der Artikel eine andere primäre Funktion haben muss. Für die Funktion als Genusmarker sprechen insbesondere die in den „Anna-Daten“ vorgefundenen Strategien zur Genusmarkierung im Genitiv. Teilnehmende der Online-Umfrage bilden Mischkonstruktionen, in denen mittels -s-Suffix am Namen ein Genitiv angezeigt wird, die aber einen onymischen Artikel aus dem Dativ oder Nominativ/Akkusativ tragen (em Vrenis Huus bzw. d Annas Teddy). Bezüglich Kasus sind diese Konstruktionen uneindeutig, dafür geben sie im Gegensatz zum Gentiv-Artikel $d s$, der in allen drei Genera Verwendung findet, ein eindeutiges Genus wieder. Sie verhindern ausserdem, dass das genitivische $d s$ vor Frauennamen als Neutrum missinterpretiert wird (was es sprachhistorisch formal ja war). Dass $d s$ im Genitiv als Neutrum angesehen wird, zeigt sich auch darin, dass der diminuierte und damit häufiger neutral verwendete Name Vreni verglichen mit den nicht diminuierten Namen am häufigsten in den Genitiv gesetzt wird. Er scheint in der Kombination mit dem Genitivartikel $d s$ breiter akzeptiert zu sein als die anderen Namen. Des Weiteren stützen die aus der Forschungsliteratur gewonnenen Erkenntnisse, dass Männernamen häufiger ohne Artikel gebraucht werden als Frauennamen, diese These der primären Funktion des Artikels als Genusmarker. Verschiedene Grammatiken geben an, dass auf Männer gemeinhin mit dem Maskulinum Bezug genommen wird (es sei denn, es steht ausdrücklich der neutrale Artikel), während auf Frauen ausnahmslos oder teilweise mit dem Neutrum verwiesen wird. Daraus folgt, dass bei Männern das sexus- 
kongruente Genus nicht explizit mit Artikelsetzung ausgedrückt werden muss, während bei Frauen die (mögliche) Inkongruenz von neutralem Genus mit dem weiblichen Sexus durch den Artikel angezeigt werden will. Diese These konnte mit den „Anna-Daten“, in denen fast ausschliesslich Frauennamen abgefragt worden sind, nicht überprüft werden. Aber eine andere Erkenntnis kann damit in Verbindung gebracht werden: Die „Anna-Daten“ zeigen, dass sich die onymische Artikellosigkeit ausbreitet und in einem sehr viel grösseren Gebiet vorkommt, als sie in der Forschungsliteratur ausgewiesen ist. Gleichzeitig zeigt sich bereits in der Forschungsliteratur und auch in den „Anna-Daten“, dass der Gebrauch des neutralen Genus für Frauen rückläufig ist. Viele Teilnehmende schreiben auch explizit in Kommentaren in der Online-Umfrage, dass sie das neutrale Genus für Frauen absichtlich vermeiden. So schreibt etwa Teilnehmerin A337: „Ich entscheide mich oft bewusst gegen die s-Form bei Frauen, auch wenn mir diese bei gewissen Frauennamen geläufiger ist. (Bethli, Trudi, Heidi, Vreni..). Die sächliche Form gibt mir das Gefühl, die Person nicht ganz als voll zu nehmen." Bei den Frauennamen stimmt das Genus zunehmend ebenfalls mit dem Sexus der Trägerin überein, das abweichende neutrale Genus ist gar nicht mehr möglich und muss darum nicht mehr mit dem Artikel angezeigt werden. Der Artikel wird damit obsolet.

Die vorliegende Untersuchung basiert auf einer interindividuellen Analyse der „Anna-Daten“, eine intraindividuelle Analyse konnte nicht geleistet werden. Da ein erster Blick in einzelne Datensätze aus der „Anna-Umfrage“ aber unter anderem zeigt, dass Artikellosigkeit zwar häufig ist, aber keine einzige Teilnehmerin durchweg alle Namen ohne Artikel gebraucht, wäre eine solche vielversprechend. Damit könnte den Fragen nachgegangen werden, an welchen Stellen Artikel weggelassen und wo sie gesetzt werden, woraus weiterführende Erkenntnisse zur Funktion des onymischen Artikels gewonnen werden könnten.

\section{Literaturverzeichnis}

Bachmann, Albert (ed.) (1910-1941): Beiträge zur Schweizerdeutschen Grammatik (BSG). 20 Bände. Frauenfeld: Huber und co.

Baumgartner, Gerda/Christen, Helen: „Dr Hansjakobli und ds Babettli - Über die Geschlechtstypik diminuierter Rufnamen in der Deutschschweiz“. In: Reisigl, Martin/Spieß, Constanze (eds.): Sprache und Geschlecht. Band 2. Duisburg, Universitätsverlag Rhein-Ruhr: 111-145. (= Osnabrücker Beiträge zur Sprachtheorie 91).

Behaghel, Otto (1923): Deutsche Syntax. Eine geschichtliche Darstellung. Band I: Die Wortklassen und Wortformen. o. O.: Carl Winter's Universitätsbuchhandlung. (= Sammlung germanischer Elementar- und Handbücher 10).

Bösiger, Melanie (2017): Evelyne redt mit s Claudias Ma. Artikellosigkeit und Artikelgebrauch vor (weiblichen) Vornamen im Schweizerdeutschen in allen vier Kasus. Universität Freiburg i. Ü.: unveröffentlichte Masterarbeit.

Brandstetter, Renward (1904): Der Genitiv der Luzerner Mundart in Gegenwart und Vergangenheit. Zürich: Zürcher \& Furrer. (= Abhandlungen herausgegeben von der Gesellschaft für deutsche Sprache in Zürich X).

Bucheli Berger, Claudia (2006): „Syntaktische Raumbilder im Höchstalemannischen“. In: Klausmann, Hubert (ed.): Raumstrukturen im Alemannischen. 15. Arbeitstagung zur alemannischen Dialektologie auf Schloss Hofen, Lochau (Vorarlberg), 19.-21.9.2005. Graz/Feldkirch, Wolfgang Neugebauer Verlag: 91-95. 
Dauwalder, Hans (1992): Haslitiitsch. Wie mma s seid und cha schriiben. Eine haslideutsche Kurzgrammatik. Meiringen: Gemeinnütziger Verein Meiringen.

Elspaß, Stephan/Möller, Robert (2012): Atlas zur deutschen Alltagssprache. Karten zur Namensnennung. Universität Salzburg. atlas-alltagssprache.de/r10-f16ab [27.07.2017].

Fischer, Ludwig (1960): Luzerndeutsche Grammatik. Ein Wegweiser zur guten Mundart. Zürich: Schweizer Spiegel Verlag. (= Grammatiken und Wörterbücher des Schweizerdeutschen II).

Fleischer, Jürg/Schallert, Oliver (2011): Historische Syntax des Deutschen. Eine Einführung. Tübingen: Narr Francke Attempto Verlag.

Gallmann, Peter (1999): Zur Morphosyntax der Eigennamen im Deutschen. Schaffhausen/Zürich: o. Verl.

Glaser, Elvira (2008): „Syntaktische Raumbilder“. In: Ernst, Peter/Patocka, Franz (eds.): Dialektgeographie der Zukunft. Akten des 2. Kongresses der Internationalen Gesellschaft für Dialektologie des Deutschen (IGDD) am Institut für Germanistik der Universität Wien, 20. bis 23. September 2006. München, Franz Steiner Verlag: 85-107.

Goldener, Hans (1908): Die Verbalflexion einer ostschweizerischen Dialektgruppe mit besonderer Berücksichtigung der Analogiebildungen. Handschriftliches Manuskript einsehbar im Archiv des schweizerischen Idiotikons.

Hodler, Werner (1969): Berndeutsche Syntax. Bern: Francke Verlag.

Hotzenköcherle, Rudolf (1984): Die Sprachlandschaften der deutschen Schweiz. Aarau: Sauerländer. (= Sprachlandschaft 1).

Kolde, Gottfried (1989): Der Artikel in deutschen Sachverhaltsnominalen. Tübingen: Max Niemeyer Verlag.

Kolde, Gottfried (1995): „Namengrammatik“. In: Eichler, Ernst et al. (eds.): Namenforschung. Ein internationales Handbuch zur Onomastik. Berlin, De Gruyter: 400-408. (= Handbücher zur Sprach- und Kommunikationswissenschaft (HSK) 11/1).

Köpcke, Klaus Michael/Zubin, David A. (1984): „Sechs Prinzipien für die Genuszuweisung im Deutschen: Ein Beitrag zur natürlichen Klassifikation“. Linguistische Berichte 93: 26-50.

Marti, Werner (1985): Berndeutsch-Grammatik für die heutige Mundart zwischen Thun und Jura. Bern: A. Francke.

Meyer, Rudolf (1967): Zur Morphologie und Sprachegeographie des Artikels im Schweizerdeutschen. Frauenfeld: Huber \& co. (= Beiträge zur schweizerdeutschen Mundartforschung (BSM) XIII).

Nübling, Damaris/Fahlbusch, Fabian/Heuser, Rita (2012): Namen. Eine Einführung in die Onomastik. Tübingen: Narr Verlag.

Schmid, Martin/Issler, Gaudenz (1982): Davoserdeutsches Wörterbuch. Der Wortschatz einer Bündner Walsermundart. Chur: Walservereinigung Graubünden. (= Grammatiken und Wörterbücher des Schweizerdeutschen VII).

Schmuck, Mirjam/Szczepaniak, Renata (2014): „Der Gebrauch des Definitartikels vor Familien- und Rufnamen im Frühneuhochdeutschen aus grammatikalisierungstheoretischer Perspektive“. In: Debus, Friedhelm/Heuser, Rita/Nübling, Damaris (eds.): Lingusitik der Familiennamen. Hildesheim, Georg Olms Verlag AG: 97-137. (= Germanistische Linguistik 225-227). 
Schmutz, Christian/Haas, Walter (2004): Senslerdeutsches Wörterbuch. Mundartwörterbuch des Sensebezirks im Kanton Freiburg mit Einschluss der Stadt Freiburg und der Pfarrei Gurmels. 2., ergänzte und korrigierte Auflage. Freiburg i. Ü.: Paulusverlag.

Schweizerisches Idiotikon. Wörterbuch der schweizerdeutschen Sprache. Frauenfeld: Huber (1881-2012)/Basel: Schwabe (2013-).

Seiler, Guido (2003): Präpositionale Dativmarkierung im Oberdeutschen. Wiesbaden: Franz Steiner Verlag. (= Zeitschrift für Dialektologie und Linguistik - Beihefte 124).

Sprachatlas der deutschen Schweiz (SDS) (1962-97). Begründet von Heinrich Baumgartner und Rudolf Hotzenköcherle; ed. von Rudolf Hotzenköcherle sowie Robert Schläpfer, Rudolf Trüb, Paul Zinsli. 8 Bde. Bern/Basel: Francke.

Suter, Rudolf (1992): Baseldeutsch-Grammatik. 3., überarbeitete Auflage. Basel: Christoph Merian Verlag. (= Grammatiken und Wörterbücher des Schweizerdeutschen VI).

Weber, Albert (1948): Zürichdeutsche Grammatik. Ein Wegweiser zur guten Mundart. Zürich: Schweizer Spiegel Verlag. (= Grammatiken und Wörterbücher des Schweizerdeutschen I). 